\title{
Interactions of Silicate Ions with Zinc(II) and Aluminum(III) in
}

\section{Alkaline Aqueous Solution}

Michel R. Anseau, Jennifer P. Leung, Nita Sahai and Thomas W. Swaddle*

\section{Supporting Information}

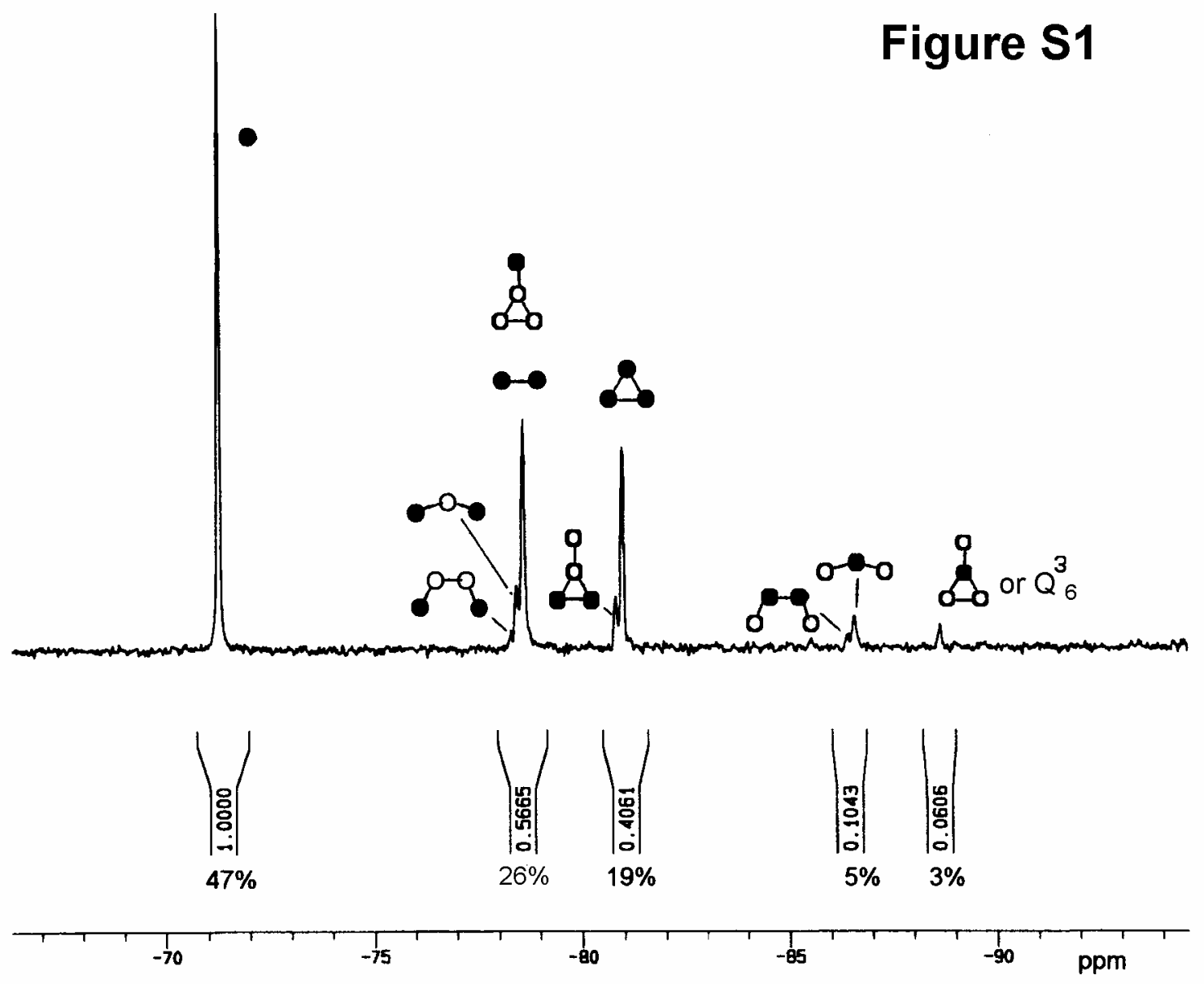

Figure S1. ${ }^{29} \mathrm{Si}$ spectrum of $1.69 \mathrm{~mol} \mathrm{~L}^{-1} \mathrm{SiO}_{2}$ dissolved in $6.4 \mathrm{~mol} \mathrm{~L}^{-1} \mathrm{KOH}$ (i.e., $1.69 \mathrm{~mol} \mathrm{~L}^{-1}$ $\mathrm{Si}(\mathrm{OH})_{2} \mathrm{O}_{2}{ }^{2-}$ and $\left.3.0 \mathrm{~mol} \mathrm{~L}^{-1} \mathrm{OH}^{-}\right)$at $280 \mathrm{~K}$. Circles in skeleton structures represent $\mathrm{Si}$ atoms; filled circles represent positions of ${ }^{29} \mathrm{Si}$ nuclei giving rise to the peak indicated. 
Figure S2
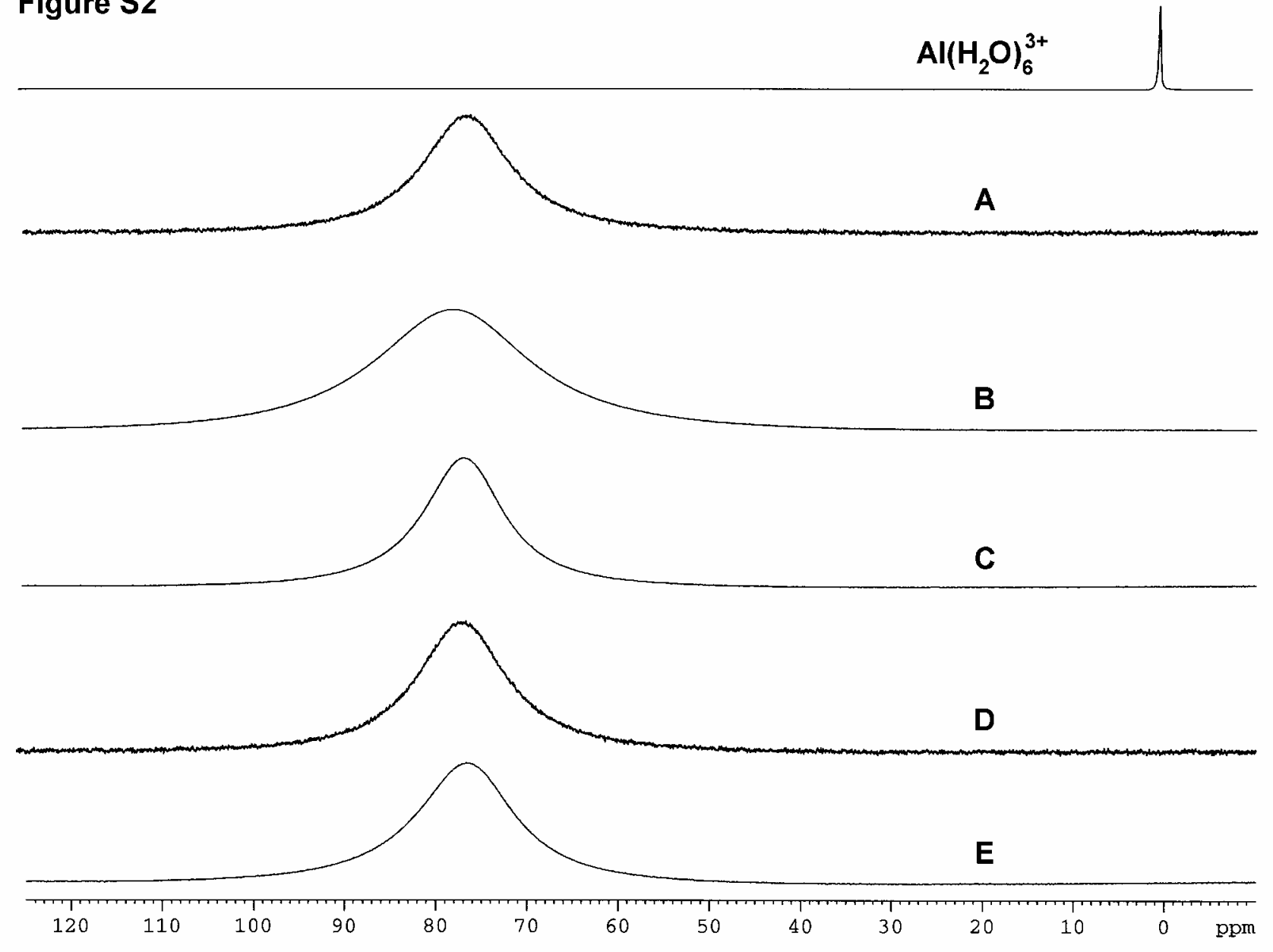

Figure S2. ${ }^{27} \mathrm{Al}$ spectra of solutions of $\mathrm{Al}$ in $15.0 \mathrm{~mol} \mathrm{~kg}{ }^{-1} \mathrm{NaOH}$ at $298 \mathrm{~K}$, relative to $\delta\left({ }^{27} \mathrm{Al}\right)=0$ for $0.56 \mathrm{~mol} \mathrm{~L}^{-1}$ aqueous $\mathrm{AlCl}_{3}$ (top spectrum); details are given in Table 2. 

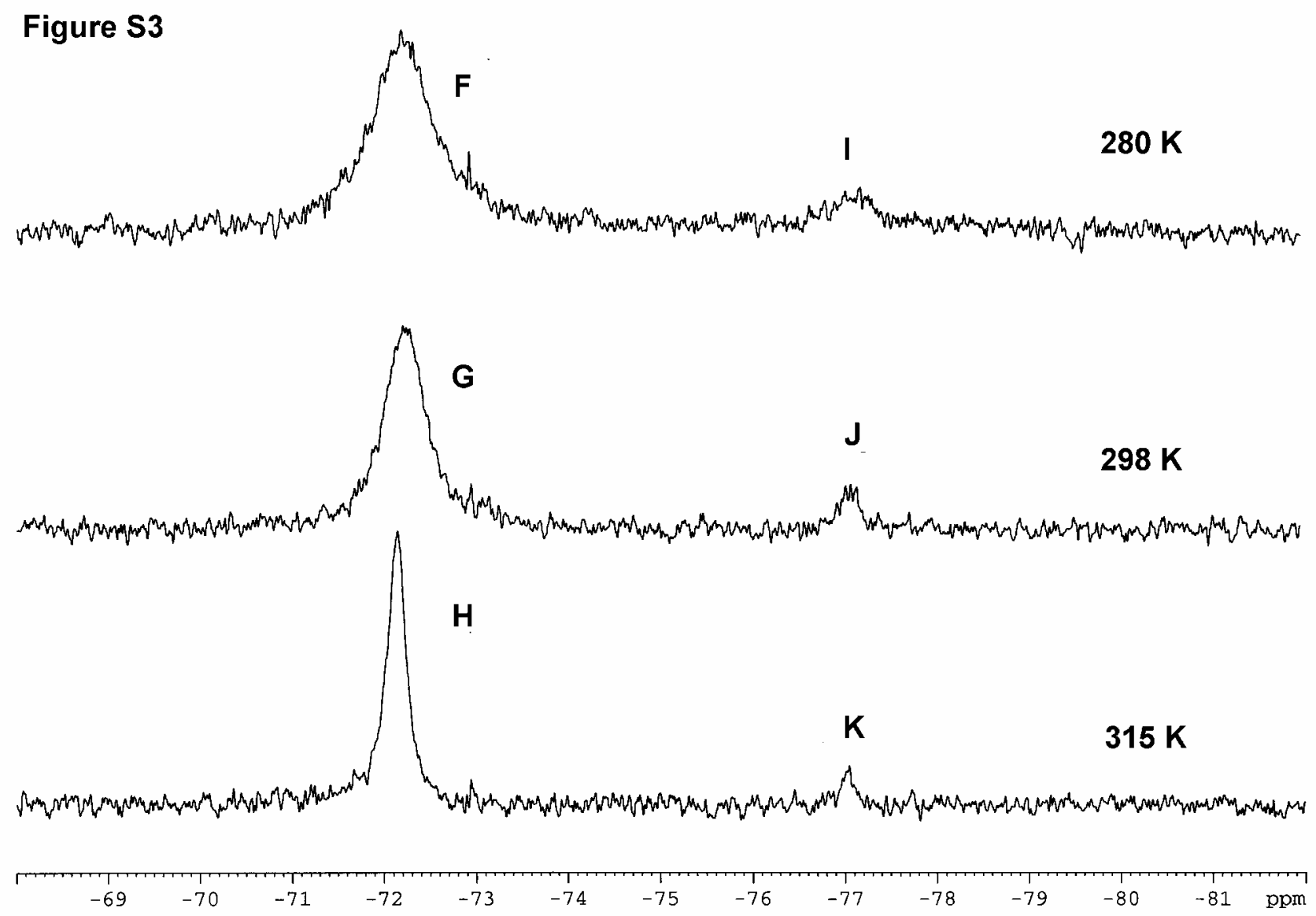

Figure S3. ${ }^{29} \mathrm{Si}$ NMR spectra of a solution containing $15.0 \mathrm{~mol} \mathrm{~kg}^{-1} \mathrm{NaOH}, 0.59 \mathrm{~mol} \mathrm{~kg}^{-1} \mathrm{Si}^{\mathrm{IV}}, 0.61 \mathrm{~mol}$ $\mathrm{kg}^{-1} \mathrm{Zn}^{\mathrm{II}}$, and $0.58 \mathrm{~mol} \mathrm{~kg}{ }^{-1} \mathrm{Al}^{\mathrm{III}}$ at 280,298 , and $315 \mathrm{~K}$. For the larger peaks $\mathrm{F}, \mathrm{G}$ and $\mathrm{H}, \delta\left({ }^{29} \mathrm{Si}\right)=-$ 72.2, -72.2, and $-72.1 \mathrm{ppm}$, with line widths 43, 31 and $13 \mathrm{~Hz}$, respectively. For peaks I, J and $\mathrm{K}$, the corresponding data are $\delta\left({ }^{29} \mathrm{Si}\right)=-77.2,-77.1$, and $-77.0 \mathrm{ppm}$, with line widths 30,14 , and $8.2 \mathrm{~Hz}$. 
Table S1. ${ }^{29}$ Si Chemical Shifts $\delta$ and Line Widths $W$ Relevant to the Calculation of the Rate Constant $k$ for the Exchange of $\left[(\mathrm{HO})_{3} \mathrm{ZnOSiO}_{2} \mathrm{OH}\right]^{4-}$ (" $\mathrm{Zn")} \mathrm{with}\left[(\mathrm{HO})_{3} \mathrm{AlOSiO}_{2} \mathrm{OH}\right]^{3-}$ (" $\mathrm{Al}$ ") at Variable Temperature According to Eq $4 .^{a}$

\begin{tabular}{|l|l|l|l|}
\hline Temp./K & 280 & 298 & 315 \\
\hline$\delta_{\mathrm{Zn}} / \mathrm{ppm}$ & -69.9 & -69.7 & -69.5 \\
\hline$\delta_{\mathrm{Al} /} / \mathrm{ppm}$ & -75.3 & -75.0 & -74.8 \\
\hline$\delta_{\mathrm{AlZn}} / \mathrm{ppm}$ & -72.2 & -72.2 & -72.1 \\
\hline$C_{\mathrm{Si}} / \mathrm{mol} \mathrm{kg}^{-1}$ & 0.515 & 0.539 & 0.536 \\
\hline$f_{\mathrm{Zn}}{ }^{b}$ & 0.570 & 0.520 & 0.505 \\
\hline$f_{\mathrm{Al}}{ }^{b}$ & 0.430 & 0.480 & 0.495 \\
\hline$\Delta v / \mathrm{Hz}$ & 326 & 319 & 313 \\
\hline$W_{\mathrm{Zn}} / \mathrm{Hz}$ & 2.6 & 3.5 & 3.6 \\
\hline$W_{\mathrm{Al} /} / \mathrm{Hz}$ & 40.4 & 12.8 & 5.7 \\
\hline$W_{\mathrm{ZnAl} /} / \mathrm{Hz}$ & 43.3 & 30.6 & 12.8 \\
\hline$k / 10^{4} \mathrm{~kg} \mathrm{~mol}^{-1} \mathrm{~s}^{-1}$ & $2.6^{c}$ & $2.6^{c}$ & 7.0 \\
\hline$k / 10^{4} \mathrm{~kg} \mathrm{~mol}^{-1} \mathrm{~s}^{-1}$ & $1.6^{d}$ & $2.3^{c}$ & 7.0 \\
\hline
\end{tabular}

${ }^{a}[\mathrm{NaOH}]=15.0 \mathrm{~mol} \mathrm{~kg}{ }^{-1} ;[\mathrm{Si}]_{\text {total }}=0.591 \mathrm{~mol} \mathrm{~kg}^{-1} ;[\mathrm{Zn}]_{\text {total }}=0.613 \mathrm{~mol} \mathrm{~kg}^{-1} ;[\mathrm{Al}]_{\text {total }}=0.578 \mathrm{~mol}$ $\mathrm{kg}^{-1}$. ${ }^{b}$ From chemical shifts. ${ }^{c}$ Using the tabulated $W_{\mathrm{Al}} .{ }^{d}$ Using $W_{\mathrm{Al}}=5.7 \mathrm{~Hz}$ at all temperatures to show impact of this quantity. 
Table S2. ${ }^{29}$ Si Chemical Shifts $\delta$ and Line Widths $W$ Relevant to the Calculation of the Rate Constant $k$ for the Exchange of $\left[(\mathrm{HO})_{3} \mathrm{ZnOSiO}_{2} \mathrm{OH}\right]^{4-}$ (" $\mathrm{Zn")} \mathrm{with}\left[(\mathrm{HO})_{3} \mathrm{AlOSiO}_{2} \mathrm{OH}\right]^{3-}$ ("Al”) at Variable Composition According to $\mathrm{Eq} 4 .^{a}$

\begin{tabular}{|l|l|l|l|l|}
\hline$[\mathrm{Si}]_{\text {total }} / \mathrm{mol} \mathrm{kg}^{-1}$ & 0.559 & 0.591 & 0.575 & 0.567 \\
\hline$C_{\mathrm{Si}} / \mathrm{mol} \mathrm{kg}^{-1}$ & 0.505 & 0.538 & 0.519 & 0.511 \\
\hline$[\mathrm{Zn}]_{\text {total }} / \mathrm{mol} \mathrm{kg}^{-1}$ & 0.306 & 0.613 & 0.710 & 0.865 \\
\hline$[\mathrm{Al}]_{\text {total }} / \mathrm{mol} \mathrm{kg}^{-1}$ & 0.817 & 0.578 & 0.417 & 0.311 \\
\hline$f_{\mathrm{Zn}}{ }^{b}$ & 0.333 & 0.520 & 0.611 & 0.685 \\
\hline$f_{\mathrm{Al}}{ }^{b}$ & 0.667 & 0.480 & 0.389 & 0.315 \\
\hline$\delta_{\mathrm{AlZn}} / \mathrm{ppm}$ & -73.2 & -72.2 & -71.7 & -71.3 \\
\hline$W_{\mathrm{AlZn}} / \mathrm{Hz}$ & 32.8 & 30.6 & 22.9 & 18.7 \\
\hline$k / 10^{4} \mathrm{~kg} \mathrm{~mol}^{-1} \mathrm{~s}^{-1 c}$ & 2.4 & 2.6 & 3.7 & 4.4 \\
\hline$k / 10^{4} \mathrm{~kg} \mathrm{~mol}^{-1} \mathrm{~s}^{-1 d}$ & 2.0 & 2.3 & 3.2 & 3.7 \\
\hline
\end{tabular}

${ }^{a}$ At $298 \mathrm{~K} ;[\mathrm{NaOH}]=15 \mathrm{~mol} \mathrm{~kg}{ }^{-1} ; \delta_{\mathrm{Zn}}=-69.65 \mathrm{ppm} ; \delta_{\mathrm{Al}}=75.0 \mathrm{ppm} ; \Delta v=319 \mathrm{~Hz} ; W_{\mathrm{Zn}}=3.5 \mathrm{~Hz}$. ${ }^{b}$ From chemical shifts. ${ }^{c} W_{\mathrm{Al}}=12.8 \mathrm{~Hz}$ (measured, $298 \mathrm{~K}$ ). ${ }^{d} W_{\mathrm{Zn}}=5.7 \mathrm{~Hz}$ (as for $315 \mathrm{~K}$ ). 
Complete reference (32): Frisch, M. J.; Trucks, G. W.; Schlegel, H. B.; Scuseria, G. E.; Robb, M. A.; Cheeseman, J. R.; Zakrzewski, V. G.; Montgomery, J. A., Jr.; Stratmann, R. E.; Burant, J. C.; Dapprich, S.; Millam, J. M.; Daniels, A.D.; Kudin, K. N.; Strain, M. C.; Farkas, O.; Tomasi, J.; Barone, V.; Cossi, M.; Cammi, R.; Mennucci, B.; Pomelli, C.; Adamo, C.; Clifford, S.; Ochterski, J.; Petersson, G. A.; Ayala, P. Y.; Cui, Q.; Morokuma, K.; Malick, D. K.; Rabuck, A. D.; Raghavachari, K.; Foresman, J. B.; Cioslowski, J.; Ortiz, J. V.; Baboul, A. G.; Stefanov, B. B.; Liu, G.;Liashenko, A.; Piskorz, P.; Komaromi, I.; Gomperts, R.; Martin, R. L.; Fox, D. J.; Keith, T.; Al-Laham, M. A.; Peng, C. Y.; Nanayakkara, A; Challacombe, M.; Gill, P. M. W.; Johnson, B.; Chen, W.; Wong, M. W.; Andres, J. L.; Gonzalez, C.; Head-Gordon, M.; Replogle, E. S.; Pople, J. A. Gaussian 98, Gaussian Inc.: Pittsburgh, PA, 1998. 